\title{
Sequential Monte Carlo methods for Discharge Time Prognosis in Lithium-Ion Batteries
}

\author{
Marcos E. Orchard ${ }^{1}$, Matías A. Cerda ${ }^{1}$, Benjamín E. Olivares ${ }^{1}$, and Jorge F. Silva ${ }^{1}$ \\ ${ }^{1}$ Department of Electrical Engineering, Universidad de Chile, Santiago, 8370451, Chile \\ morchard@ing.uchile.cl \\ matias.cm.uc@gmail.com \\ benjamin.olivares.r@gmail.com \\ josilva@ing.uchile.cl
}

\begin{abstract}
This paper presents the implementation of a particlefiltering-based prognostic framework that allows estimating the state-of-charge (SOC) and predicting the discharge time of energy storage devices (more specifically lithium-ion batteries). The proposed approach uses an empirical statespace model inspired in the battery phenomenology and particle-filtering to study the evolution of the SOC in time; adapting the value of unknown model parameters during the filtering stage and enabling fast convergence for the state estimates that define the initial condition for the prognosis stage. SOC prognosis is implemented using a particlefiltering-based framework that considers a statistical characterization of uncertainty for future discharge profiles.
\end{abstract}

\section{INTRODUCTION}

Energy storage devices (ESDs) play a key role in both industrial and military machinery. Consider, for example, electronic products commonly associated with daily living (laptop computers, communications equipment, GPS, domestic robots) and some other more sophisticated pieces of equipment and machinery such as pacemakers ground and aerial vehicles (manned, tele-commanded and unmanned), and satellites. ESDs may be used as primary sources of energy or as backup, allowing energizing different devices and systems under various operating profiles; thus improving their autonomy.

Particularly nowadays, Lithium-based compounds have presented significant advantages over other chemical combinations, such as Ni-MH, Ni-Cd and lead, when used for the manufacture of ESDs. In part this is explained by the fact that Li-Ion ESDs offer larger charge density by unit of

Marcos E. Orchard et al. This is an open-access article distributed under the terms of the Creative Commons Attribution 3.0 United States License, which permits unrestricted use, distribution, and reproduction in any medium, provided the original author and source are credited. mass (or volume), allowing to design very compact cells that can be easily integrated to small electronic devices; in addition, Li-Ion batteries offer extended life cycles and limited self-discharge rates (Saha and Goebel, 2009; Ranjbar et al., 2012). Due to the exponential increase in the use of Li-Ion ESDs within the automotive industry, and the projected demand associated to this type of vehicles, the concept of "Battery Management Systems" - BMS, (Pattipati et al., 2011) - started to become more a necessity than a luxury. These systems have as main objective (i) to provide and maximize usage time (autonomy) that is associated to a discharge cycle, (ii) to reduce battery charging times, (iii) to maximize the number of operating cycles for the ESD, and (iv) real-time operation, adjusting to sudden changes in charge/discharge conditions. To achieve this, BMS must consider at least information about the battery "state-of-charge" - SOC (Pattipati et al., 2011), the "state-of-health" - SOH (Pattipati et al., 2011), and the "remaining useful life" - RUL (Orchard and Vachtsevanos, 2009) of cells within the pack. On the one hand, knowledge about the SOC will help to quantify the autonomy of the system (based on some assumptions on the future discharge profile). On the other hand, knowledge about the $\mathrm{SOH}$ or the RUL of specific cells within the battery pack could help to decide when to replace or recycle it.

This article focuses on the problem of estimating and predicting the SOC of a Li-Ion ESD. Given that several definitions for the concept of SOC can be found in literature, it is relevant to mention that this research effort defines the SOC as the remnant energy (measured as a percentage of the current maximum cell capacity) in the battery. The SOC is highly affected by charge/discharge rate, temperature, hysteresis effects, usage time and selfdischarge (due to the internal resistance of the cell), which transforms this particular prognosis problem into a very challenging one. Three specific aspects have to be carefully considered when intending to implement an efficient (and 
effective) SOC prognosis approach: (i) how to model the battery, (ii) how to estimate the SOC in a nonlinear, nonobservable system, and (iii) how to predict the impact of future discharge profiles in the evolution of SOC in time. Several research efforts aim at providing a solution for the first two open questions, using empirical, physicochemical, or electric models in conjunction with estimation techniques based on fuzzy logic (Salkind et al., 1999), neural networks (Charkhgard and Farrokhi, 2010), or Bayesian approaches such as the extended Kalman filter - EKF (Saha and Goebel, 2009; Vinh Do et al., 2009).

This work has selected a combination of an empirical statespace model inspired in the battery phenomenology and Bayesian filtering techniques to study the evolution of the SOC in time; adapting and learning the value of unknown model parameters during the filtering stage, and enabling fast convergence for the state estimates that define the initial condition for the SOC prognosis stage. Due to model nonlinearities and the existence of non-Gaussian noise sources, the proposed approach considers the use of sequential Monte Carlo methods in prognosis, a sub-optimal filtering technique also known as particle-filtering. SOC prognosis is implemented using a particle-filtering-based framework (Orchard and Vachtsevanos, 2009) that considers uncertainty in the future discharge profile by including several possible future scenarios in the computation of the discharge time probability density function (PDF).

The structure of the article is as follows. Section 2 presents a theoretical framework for the problem of SOC estimation and prognosis, as well as failure prognosis using sequential Monte Carlo methods. Section 3 focuses on the modeling aspects that are required to incorporate the impact of different discharge profiles on the battery SOC. Section 4 shows the obtained results for SOC prognosis when trying to estimate the discharge time of a Li-Ion battery that energizes a ground robot. Finally, Section 5 presents the main conclusions of this research effort.

\section{THEORETICAL FrAMEWORK}

\subsection{State-of-Charge Estimation in Lithium-Ion Batteries}

The state-of-charge provides an indicator of the system autonomy that directly depends on the remaining battery energy and the mission profile; a critical piece of information for the design of path planning/control strategies in autonomous vehicles. It is for this reason that the implementation of SOC estimation and prognostic algorithms (Saha and Goebel, 2009; Ranjbar et al., 2012; Pattipati et al., 2011; Salkind et al., 1999; Charkhgard and Farrokhi, 2010; Vinh Do et al., 2009; Ran et al., 2010; Cadar et al., 2009; Qingsheng et al., 2010; Di et al., 2011; Tang et al., 2011) is considered the first step towards online characterization of both the End-of-Discharge (EoD) time and RUL of Li-Ion batteries. One of the main difficulties in SOC estimation is that it cannot be measured directly, and thus its value must be inferred from the observation of other variables such as the battery current, voltage, temperature, state-of-health degradation and self-discharge phenomena (Pattipati et al., 2011; Cadar et al., 2009; Qingsheng et al., 2010; Di et al., 2011). Indeed, the utilization of more complex electrochemical models has been only suitable for off-line studies, mainly because these models (i) require a large number of variables to represent the battery internal structure, (ii) assume extremely accurate measurements, and (iii) have an elevated computational cost (Pattipati et al., 2011; Charkhgard and Farrokhi, 2010). Other options for SOC monitoring include the open-circuit voltage (OCV) method. This approach has the advantage of providing a direct relationship between battery SOC and voltage measurements - the higher the OCV, the higher the SOC (Tang et al., 2011). Unfortunately, the implementation of this test requires large resting periods for the battery, limiting its use for online applications (Pattipati et al., 2011; Charkhgard and Farrokhi, 2010; Di et al., 2011; Tang et al., 2011). Similarly, the "Electrochemical Impedance Spectroscopy” (EIS) (Pattipati et al., 2011; Ran et al., 2010) is a noninvasive method that intends to provide a complete characterization of the battery internal equivalent circuit. However, the implementation of an EIS test requires the acquisition of costly equipment (generally found only at laboratory test sites), which severely limits its widespread use in practice (Dalal et al., 2011). It is for this reason that current research efforts for SOC estimation and prognostic algorithms have focused on approaches that are mostly based on empirical models that incorporate only critical phenomenological aspects of the process; i.e., the relationship between currents, voltages and temperatures of Li-Ion cells. Among these methods, it is worth mentioning those that are based on fuzzy logic, neural networks, and Bayesian approaches.

On the one hand, fuzzy logic models have been used for the SOC estimation either through the identification of equivalent circuit for the battery from EIS data or directly from voltage and current measurements (Salkind et al., 1999). Given that EIS data have proved to be very noisy in practice (Saha et al., 2009; Dalal et al., 2011), only the latter case represents a reasonable method for online SOC estimation and uncertainty characterization. However, even in that case, the problem of SOC prediction (related to battery prognosis) is still unresolved and mainly treated as a curve regression problem (which is insufficient for purposes of risk characterization). Neural networks have also been used to build a nonlinear relationship between battery measurements and the evolution of SOC in time (Pattipati et al., 2011; Charkhgard and Farrokhi, 2010; Qingsheng et al., 2010). These methods, however, do not provide an adequate representation for uncertainty in nonlinear systems and thus neither can they be used for risk quantification purposes. 
On the other hand, recent years have seen a growing interest in the use of machine learning techniques (e.g., Hamming Networks (Lee et al., 2011)) and stochastic filtring techniques (unscented Kalman Filter (Santhanagopalan and White, 2010), extended Kalman Filter (Hu et al., 2012), and unscented particle filter (He et al., 2013)) to estimate the SOC and/or parameter degradation of a Li-Ion battery cell under a randomly varying loading condition. Suboptimal Bayesian methods have proven particularly effective in the task of simultaneously incorporate information from noisy measurements and characterize the sources of uncertainty (Charkhgard and Farrokhi, 2010; Vinh Do et al., 2009; Di et al., 2011; Saha et al., 2009; Dalal et al., 2011; Orchard et al., 2010). In fact, experience has demonstrated that Bayesian state estimators are especially well suited for realtime estimation problems associated to dynamic state models (Saha et al., 2009; Dalal et al., 2011; Orchard et al., 2010). In addition, these methods also provide a concrete characterization of uncertainty sources both in the filtering and the prediction stage, a piece of information that is required for the generation of a risk measure associated to SOC prognosis. Bayesian estimators require a state-space model for the dynamic system, and prognostic modules based on a state-space formulation for the dynamic system are very sensitive to the initial condition of the state vector. For this reason, the implementation of accurate online SOC estimators is absolutely relevant for the development of real-time predictors capable of quantifying the feasibility (as well as the cost) of a particular vehicle trajectory. Depending on the validity of linear or Gaussian assumptions, either an extended Kalman filter or a particlefiltering (Arulampalam et al., 2002; Andrieu et al., 2001; Doucet et al., 2001) approach may be needed.

To evaluate which is the best option for this particular problem, it is first necessary to define a state model that represents adequately the dynamics associated to the evolution of the battery SOC, for a given usage profile. This research has considered for this purpose the problem of battery end-of-charge prognosis in a four-wheel ground robot. In this scenario, different discharge profiles can be verified due to terrain conditions (hills, surface changes) and other factors, while the robot autonomously has to perform a pre-determined mission. Given that the robot is currently configured only for use on $2 \mathrm{D}$, uniform terrain, it is necessary to simulate the environment through a variable load has been attached to the battery. This variable load is made up of three resistors $(6.23 \Omega, 12.5 \Omega$, and $25 \Omega$ ), each wired in parallel to the battery to increase current draw. Each resistor can be activated via a relay controlled by the onboard computer's data acquisition card. It provides 8 different loading scenarios progressing linearly in magnitude. The onboard computer has a map of simulated terrain and when the robot crosses into an area of higher simulated difficulty on the map, the onboard computer activates a larger loading scenario using the variable load.
This allows for many simulated terrains while keeping the robot in a safe, uniformly flat environment. Online data consists of voltage and current measurements (with the corresponding timestamp), for a lithium iron phosphate $\left(\mathrm{LiFePO}_{4}\right)$ battery $(12.8[\mathrm{~V}], 2.4[\mathrm{Ah}], 14[\mathrm{~A}]$ maximum discharge current). This experimental setup implies that for full speed $(700[\mathrm{~mm} / \mathrm{s}])$, the current drained from the battery ranges between 1.6025[A] and 5.4738[A] (depending on the value of the equivalent resistor that is connected in parallel to the battery); at $10 \%$ speed $(70[\mathrm{~mm} / \mathrm{s}])$ the current drained from the battery ranges between 0.6006[A] and 4.3971[A].

\subsection{Particle-Filtering-based Prognosis Framework for Faulty Dynamic Nonlinear Systems}

Consider a sequence of probability distributions $\left\{\pi_{k}\left(x_{0: k}\right)\right\}_{k \geq 1}$, where it is assumed that $\pi_{k}\left(x_{0: k}\right)$ can be evaluated pointwise up to a normalizing constant. Sequential Monte Carlo (SMC) methods, also referred to as particle filters (PF), are a class of algorithms designed to approximately obtain samples from $\left\{\pi_{k}\right\}$ sequentially; i.e., to generate a collection of $N \gg>1$ weighted random samples (particles) $\left\{w_{k}^{(i)}, x_{0: k}^{(i)}\right\}_{i=1 \cdots N}, w_{k}^{(i)} \geq 0, \forall k \geq 1$, satisfying (Andrieu et al., 2001; Doucet et al., 2001):

$$
\sum_{i=1}^{N} w_{k}^{(i)} \varphi_{k}\left(x_{0: k}^{(i)}\right) \underset{N \rightarrow \infty}{\longrightarrow} \int \varphi_{k}\left(x_{0: k}\right) \pi_{k}\left(x_{0: k}\right) d x_{0: k},
$$

in probability and where $\varphi_{k}$ is any $\pi_{k}$-integrable function.

In the particular case of the Bayesian Filtering problem, the target distribution $\pi_{k}\left(x_{0: k}\right)=p\left(x_{0: k} \mid y_{1: k}\right)$ is the posterior PDF of $X_{0: k}$, given a realization of noisy observations $Y_{1: k}=y_{1: k}$.

Let a set of $N$ paths $\left\{x_{0: k-1}^{(i)}\right\}_{i=1 \cdots N}$ be available at time $k-1$. Furthermore, let these paths distribute according to $q_{k-1}\left(x_{0: k-1}\right)$, also referred to as the importance density function at time $k-1$.Then, the objective is to efficiently obtain a set of $N$ new paths $\left\{\tilde{x}_{0: k}^{(i)}\right\}_{i=1 \cdots N}$ distributed according to $\pi_{k}\left(\tilde{x}_{0: k}\right)$ (Andrieu et al., 2001).

For this purpose, the current paths $x_{0: k-1}^{(i)}$ are extended by using the kernel $q_{k}\left(\tilde{x}_{0: k} \mid x_{0: k-1}\right)=\delta\left(\tilde{x}_{0: k-1}-x_{0: k-1}\right) \cdot q_{k}\left(\tilde{x}_{k} \mid x_{0: k-1}\right)$; i.e., $\tilde{x}_{0: k}=\left(x_{0: k-1}, \tilde{x}_{k}\right)$. The importance sampling procedure generates consistent estimates for the expectations of any function, using the empirical distribution (Doucet et al., 2001):

$$
\tilde{\pi}_{k}^{N}\left(x_{0: k}\right)=\sum_{i=1}^{N} w_{0: k}^{(i)} \delta\left(x_{0: k}-\tilde{x}_{0: k}^{(i)}\right)
$$

where $w_{0: k}^{(i)} \propto w_{0: k}\left(\tilde{x}_{0: k}^{(i)}\right)$ and $\sum_{i=1}^{N} w_{0: k}^{(i)}=1$. 
The most basic SMC implementation -the sequential importance sampling (SIS) particle filter- computes the value of the particle weights $w_{0: k}^{(i)}$, by setting the importance density function equal to the a priori state transition PDF $p\left(\tilde{x}_{k} \mid x_{k-1}\right)$; i.e., $q_{k}\left(\tilde{x}_{0: k} \mid x_{0: k-1}\right)=p\left(\tilde{x}_{k} \mid x_{k-1}\right)$. In that manner, the weights for the newly generated particles are evaluated from the likelihood of new observations. The efficiency of the procedure improves as the variance of the importance weights is minimized. The choice of the importance density function is critical for the performance of the particle filter scheme and hence, it should be considered in the filter design.

Prognosis (Engel et al., 2000), and thus the generation of long-term prediction, is a problem that goes beyond the scope of filtering algorithms since it involves future time horizons. Hence, if PF-based algorithms (Orchard et al., 2009; Edwards et al., 2010; Chen et al., 2011) are to be used, it is necessary to propose a procedure with the capability to project the current particle population in time in the absence of new observations.

Any adaptive prognosis scheme requires the existence of at least one feature providing a measure of the severity of the fault condition under analysis - fault dimension (Zhang et al., 2011). If many features are available, they can always be combined to generate a single signal. In this sense, it is always possible to describe the evolution in time of the fault dimension through the nonlinear state equation.

By using the aforementioned state equation to represent the evolution of the fault dimension in time, it is possible to generate $p$-ahead long term predictions, using kernel functions to reconstruct the estimate of the state PDF in future time instants, as it is shown in Eq. (3):

$$
\tilde{p}\left(x_{k+p} \mid \tilde{x}_{1: k+p-1}\right) \approx \sum_{i=1}^{N} w_{k+p-1}^{(i)} K\left(x_{k+p}-E\left[x_{k+p}^{(i)} \mid \tilde{x}_{k+p-1}^{(i)}\right]\right),
$$

where $K(\cdot)$ is a kernel density function, which may correspond to the process noise PDF, a Gaussian kernel or a rescaled version of the Epanechnikov kernel (Orchard and Vachtsevanos, 2009; Orchard et al., 2010; Orchard, Tobar and Vachtsevanos, 2009). The resulting predicted state PDF contains critical information about the evolution of the fault dimension over time. One way to represent that information is through the computation of statistics (expectations, 95\% confidence intervals), either the End-of-Discharge (EOD) (Saha et al., 2009) or the Remaining Useful Life (RUL) of the faulty system.

The EOD PDF depends on both long-term predictions and empirical knowledge about critical conditions for the system (Saxena et al., 2010; Tang, Orchard et al., 2011). This empirical knowledge is usually incorporated in the form of thresholds for main fault indicators. Therefore, the probability of failure at any future time instant $k=e o d$ (namely the EOD PDF) is given by (Orchard and Vachtsevanos, 2009):

$$
\operatorname{Pr}\{E O D=e o d\}=\sum_{i=1}^{N} \operatorname{Pr}\left(\text { Failure } \mid X=\hat{x}_{\text {eod }}^{(i)}\right) \cdot w_{\text {eod }}^{(i)} .
$$

The conditional probability of failure in Eq. (4) may be defined via the determination of hazard zones (Orchard and Vachtsevanos, 2009), either using historical data or knowledge from process operators. The simplest case is where the concept of "failure" implies the moment when the fault feature crosses a given threshold. In that case the probability of failure, conditional to the state, is equal to one if the state is exactly on the manifold that defines the threshold value.

\section{STATE-SPACE MODEL FOR StATE-OF-CHARGE ESTIMATION IN ESDS}

This article focuses on the development and implementation of a module for online discharge time prognosis in Li-Ion ESDs, using an adequate characterization of the future usage profile. For this, firstly it is necessary to model the effect that any arbitrary discharge current profile may have on the battery SOC. This model should also have a reduced number of parameters, allowing estimating and prognosticating in real-time in and accurate and precise manner. Because of these facts, physicochemical models were discarded as a feasible choice since their complexity (and the need of extremely precise off-line measurements) implied a high computational cost. Other options, such as electric equivalent circuits, with parameters that could be estimated from EIS measurements, were also discarded since (as it has been already mentioned) the implementation of such tests requires the acquisition of costly equipment, severely limiting its widespread use in practice (Dalal et al., 2011). Therefore, this research effort has chosen a grey-box (Gonzalez et al., 2003) empirical (discrete-time) model that is inspired in the battery phenomenology. The selected model only depends on voltage and discharge current measurements, something that enables its use for online prognostics modules. The proposed structure requires defining necessarily one state as the battery SOC. In addition, it is important to consider adaptation of the model to a particular battery under supervision, through the definition of a state associated to an unknown model parameter. The model used is shown below:

\section{State transition model:}

$$
\begin{gathered}
x_{1}(k+1)=x_{1}(k)+\omega_{1}(k), \\
x_{2}(k+1)=x_{2}(k)+v(k) \cdot i(k) \cdot \Delta t \cdot 10^{-5}+\omega_{2}(k),
\end{gathered}
$$

\section{Measurement equation:}

$$
\begin{gathered}
v(k)=\left[v_{0}-x_{1}(k) \cdot i(k)-e^{C \cdot x_{2}(k)}+\eta(k)\right], \\
v_{0}=13[V] ; \quad C=5.5687 ; x_{1}(0)=0.0897 ; x_{2}(0)=-E_{0}
\end{gathered}
$$


where the battery current level $i(k)[\mathrm{A}]$ and the sampling period $\Delta t[\mathrm{sec}]$ are input variables, and the battery voltage $v(k)[\mathrm{V}]$ is the system output. The states are defined as $x_{1}(k)$ (unknown model parameter) and $x_{2}(k)$ (additive inverse of SOC, remnant battery energy measured in [VA sec $\left.10^{-5}\right]$ ), $E_{0}$ is the ESD initial SOC (that could be inferred from data acquired during the charging process).

Process noises $\omega_{1}$ and $\omega_{2}$ represent uncertainty on the a priori state estimates, and $C$ is a constant that characterizes the battery voltage drop in terms of the remaining SOC. It is important to note that process noise (at least noise $\omega_{2}$ ) is correlated with $\eta$, the measurement noise, since uncertainty on battery SOC depend on the uncertainty of voltage measurements. This fact will be considered when designing the prognostic module. The state $x_{1}$ in Eq. (7) represents the instantaneous value for the battery internal resistance. It is well known that this value depends on other environmental factors (e.g., temperature). As in this case the experimental setup did not include temperature probes, then the filtering stage must infer the effect of the external temperature (and other unmeasured perturbations) into the state estimate $x_{1}$, based solely on voltage and current measurements.

Validation data (see Figure 1) for the implemented algorithms was obtained from a mobile platform developed at Impact Technologies, LCC, which basically consisted of a four-wheel ground robot used to generate different battery discharge profiles due to terrain conditions (hills, surface changes). Given that the robot is currently configured only for use on 2D, uniform terrain, it is necessary to simulate the environment through a variable load has been attached to the battery. This variable load is made up of three resistors $(6.23[\Omega], 12.5[\Omega]$, and $25[\Omega])$, each wired in parallel to the battery to increase current draw. Each resistor can be activated via a relay controlled by the onboard computer's data acquisition card. It provides 8 different loading scenarios progressing linearly in magnitude. The onboard computer has a map of simulated terrain and when the robot crosses into an area of higher simulated difficulty on the map, the onboard computer activates a larger loading scenario using the variable load. This allows for many simulated terrains while keeping the robot in a safe, uniformly flat environment.

Online data consists of voltage and current measurements (with the corresponding timestamp), for a lithium iron phosphate $\left(\mathrm{LiFePO}_{4}\right)$ battery $(12.8[\mathrm{~V}], 2.4[\mathrm{Ah}], 14[\mathrm{~A}]$ maximum discharge current). As the battery voltage drops, maximum values of the drain current may increase. Figure 1 show measured data for the battery voltage [V] and current $[\mathrm{A}]$ in an experiment where the energy accumulator was used until it discharged almost completely.

The nonlinear model proposed in Eq. (5)-(7) simultaneously allows a statistical characterization of future battery discharge profiles and the implementation of Bayesian prognostic approaches, such as those based on particle- filtering techniques (Orchard and Vachtsevanos, 2009; Saha et al., 2009; Orchard et al., 2010; Orchard, Tobar, and Vachtsevanos, 2009; Edwards et al., 2010; Chen et al., 2011; Zhang et al., 2011). Statistical characterization of discharge current profiles may incorporate information from past measurements, mainly from the ESD current $i(k)$, to understand the manner in which the ESD has been used lately. Aggressive usage profiles will translate in reduced discharge times and, conversely, low energy consumptions will lead to extended period of autonomy. Although diverse factors may affect future utilization of the ESDs, typically the system that uses those devices as primary sources of energy will operate trying to achieve similar performance indicators before and after the prognosis time; i.e., the time when long-term predictions are computed. For this reason it is critical to characterize this usage profile during the filtering stage, where the battery current $i(k)$ is an input to the estimator, and thus a known signal.

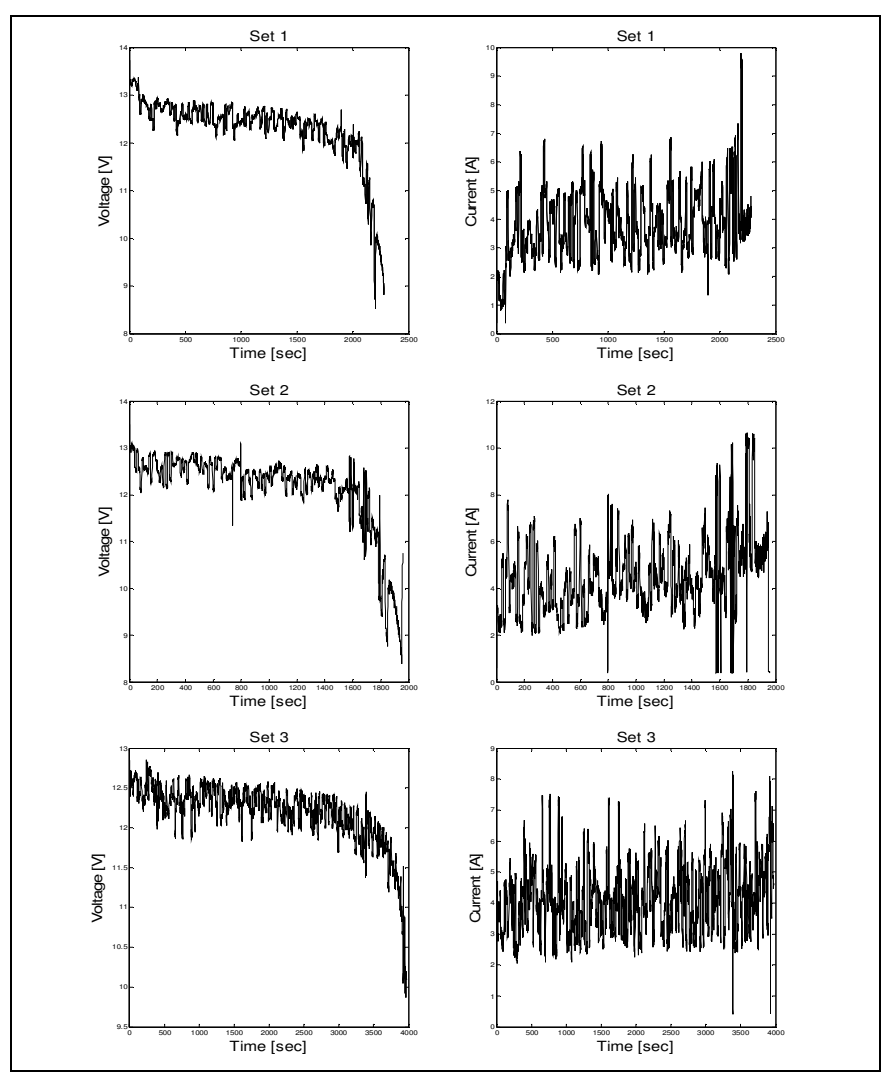

Figure 1: Measured voltage and discharge current data for a ground robot using a Li-Ion ESD. Curves on the left show voltage $[\mathrm{V}]$ as the battery discharges, while the curves on the right show the battery current $[\mathrm{A}]$.

The proposed approach for statistical characterization of battery discharge current profiles is based on a combination of algorithms that define an operating range through the computation of maximum/minimum discharge currents and empiric distributions of the acquired measurement data. The procedure used to compute extreme values is as follows: 
1. Firstly, data from the current usage profile (measured battery current) is segmented in identical time intervals, as shown in Figure 2.

2. On each interval, the maximum and minimum values for the battery current, $i_{\text {low }}^{(m)}$ and $i_{\text {high }}^{(m)}$ respectively, are computed ( $m$ is an index for the $m^{\text {th }}$ interval). These values define a range for the battery current that could be used to characterize the future operation profile of the battery, in a scenario where the prognosticator solely considers data from the $m^{\text {th }}$ time interval to this purpose. A low-pass filter was used to discard outliers and/or anomalous currents peaks.

3. An exponentially weighted moving average - EWMA (Hunter, 1986) is used to reduce the impact of an arbitrary definition for time intervals, and to incorporate prior information about battery usage. EWMA computes the extreme values that will be used to characterize the range for future battery discharge currents:

$$
\begin{aligned}
& \bar{i}_{\text {low }}^{(m)}=(1-\alpha) \cdot i_{\text {low }}^{(m)}+\alpha \cdot \bar{i}_{\text {low }}^{(m-1)} \quad \forall m, \\
& \bar{i}_{\text {high }}^{(m)}=(1-\alpha) \cdot i_{\text {high }}^{(m)}+\alpha \cdot \bar{i}_{\text {high }}^{(m-1)} \quad \forall m,
\end{aligned}
$$

where $\bar{i}_{\text {low }}^{(m)}$ and $\bar{i}_{\text {high }}^{(m)}$ are, respectively, the minimum and the maximum values that would be assumed for the future battery discharge profile, if the prediction were to be computed at the end of the $m^{\text {th }}$ interval (see Figure 2). The parameter $\alpha$ corresponds to the forgetting factor of the EWMA algorithm $(\alpha=0.65$ in this case).

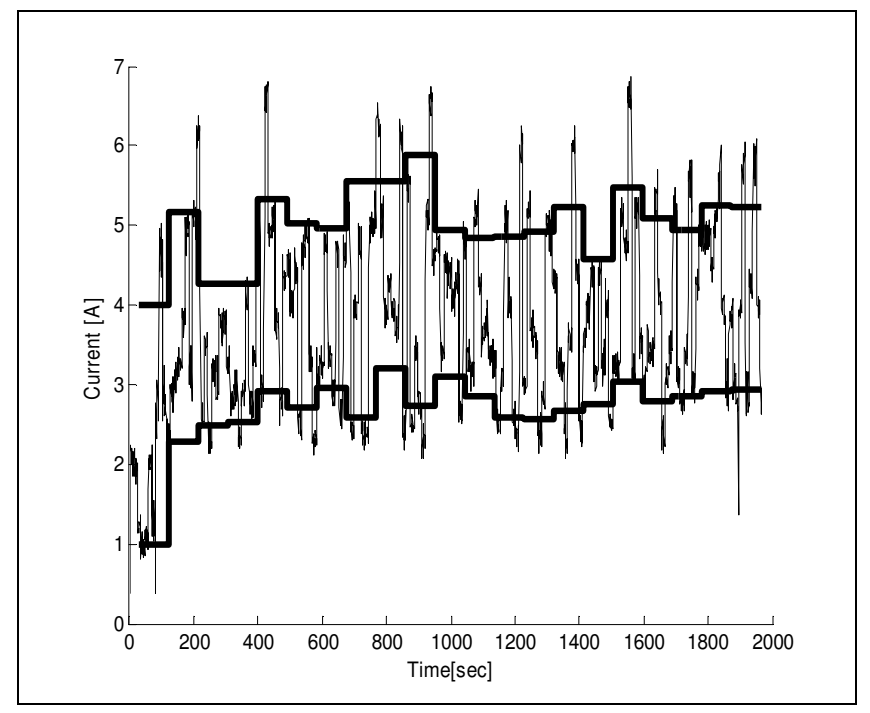

Figure 2: Characterization of maximum and minimum discharge current levels for future operation of the ESD based on exponentially weighted moving average algorithms.
The obtained range enables the generation of empirical distributions for the values that characterize the uncertainty in the future battery discharge profile; considering information about how the device has been used so far. More details on the construction of this empirical distribution will be described in the following section.

\section{Particle-filtering-Based Discharge Time PrognOSIS FOR LITHIUM-ION ESDS}

The problem of battery EOD time has been deeply discussed by several authors in the recent years (see Section 2.1). Most of them, though, intend to learn the trend of the discharge curve assuming that the only sources of uncertainty are associated to unknown model parameters or the estimates of the state vector, while the future operating profile is assumed as a deterministic function of time (constant battery current, most of the times). This article proposes a framework that intends to complement the characterization of the uncertainty associated to future discharge profiles to improve the accuracy of prognostic results, combining a classic implementation of a particlefiltering-based prognostic framework (Orchard and Vachtsevanos, 2009; Orchard et al., 2010) with a statistical characterization of the previous battery usage.

Indeed, several results (see Section 2.1) indicate that Eq. (3)-(7) can be used to show that the a priori state PDF for future time instants, and thus the EOD PDF, directly depends on the a priori probability distribution of the battery discharge profile for future time instants. Most of the times, long-term predictions assume that the latter distribution is a Dirac's delta function (a deterministic function of time for future discharge profiles). Although this simplification helps to speed up the prognostic procedure and to generate the most likely EOD estimate, it does not allow considering future changes in operating conditions or unexpected events that could affect the autonomy of the system under analysis.

Monte Carlo simulation can be used to generate EOD estimates for arbitrary a priori distributions of future operating conditions, however it is not always possible to obtain these results in real-time. In this sense, PF-based prognostic routines not only provide a theoretical framework where these concepts can be incorporated in real-time (Edwards et al., 2010), but also allow the use of uncertainty measures to characterize the sensitivity of the system with respect to changes in future load distributions (see Figure 3). Furthermore, if a formal definition of mass probability is assigned to each possible operating condition, an EOD PDF estimate can be obtained as a weighted sum of kernels, where each kernel represents the PDF estimate of a known discharge current profile, characterized as a function of time. Indeed, if the a priori distribution of future operating conditions is given by: 


$$
\operatorname{Pr}\{U=u\}=\sum_{j=1}^{N_{u}} \pi_{j} \delta\left(u-u_{j}\right),
$$

where $\left\{u_{j}\right\}_{j=1}^{N_{u}}$ is a set of deterministic time functions, then the probability of failure at a future time $t$ can be computed using Eq. (11) (Edwards et al., 2010).

$$
\operatorname{Pr}\{E O D=e o d\}=\sum_{j=1}^{N_{u}} \pi_{j} \sum_{i=1}^{N} \operatorname{Pr}\left(\text { Failure } \mid X=\hat{x}_{\text {eod }}^{(i)}, u=u_{j}\right) \cdot w_{\text {eod }}^{(i)} .
$$

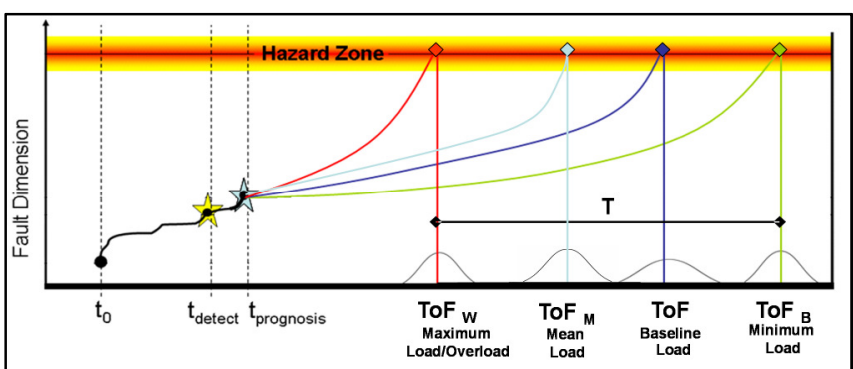

Figure 3: Illustration for the effect that different load/stress profiles may have on the growth of the fault dimension in a nonlinear dynamic system. More aggressive profiles typically result in a shorter RUL for the system.

This particular research effort considered a statistical characterization of future battery operation $(i(k)$, $k=t_{\text {pred }}+1, \ldots$, EOD) that assumed constant battery current profiles for each of the deterministic functions of time $\left\{u_{j}\right\}_{j=1}^{N_{u}}$. The probabilities $\pi_{j}$ associated to each path $u_{j}$ were computed through empirical distribution of past measurements for the battery current, and considering that the support (domain) of the empirical distribution is given by the interval $\left[\hat{i}_{\text {low }}^{-(m)}, \bar{i}_{\text {high }}^{(m)}\right]$ that is calculated using Eq. (8)-(9).

Tables 1 through 3 show the obtained results when considering $N_{u}=1,2$, and 3 respectively, computed at a particular time instant where the long-term prediction is to be calculated for model (5)-(7). For each one of these cases, the value of the future battery discharge current $u_{j}$ is computed as the center of an interval defined by the

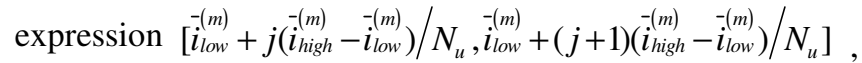
$j=0, \ldots, N_{u^{-}}-1$ In addition, once the empirical distribution is built, it is possible to compute the expectation of the future battery discharge current as a weighted average.

In this case, as the $N_{u}$ increases, it is important to note that there is only a limited impact on the probability that is assigned to the intervals that represent maximum, minimum and the median discharge currents. This fact is critical when deciding the number of paths to be considered in long-term predictions (the battery), since each prediction path involves the computation of a conditional PDF in real-time (Edwards et al., 2010). If limited computational resources are available, it may be wise to select the minimum possible number of paths that can help to represent the uncertainty of the battery operating conditions. A throughout analysis of the data presented in Tables 1 through 3 indicates that three possible discharge profiles are sufficient to characterize the tails of the EOD PDF, since the EOD increases monotonically as the battery discharge current decreases.

\begin{tabular}{|c|c|c|c|}
\hline $\boldsymbol{j}\left(\boldsymbol{N}_{\boldsymbol{u}}=\mathbf{3}\right)$ & \# Samples & $\boldsymbol{\pi}_{\boldsymbol{j}}$ & Current [A] \\
\hline $1^{\text {st }}$ & 228 & 0.4551 & 3.2965 \\
\hline $2^{\text {nd }}$ & 132 & 0.2635 & 4.3177 \\
\hline $3^{\text {rd }}$ & 141 & 0.2814 & 5.3388 \\
\hline $\begin{array}{c}\text { Weighted } \\
\text { Average }\end{array}$ & 501 & 1 & 4.1403 \\
\hline
\end{tabular}

Table 1: Empirical distributions that define the weights $\pi_{j}$ for three future battery discharge profiles in a particlefiltering-based prognosis approach.

\begin{tabular}{|c|c|c|c|}
\hline $\boldsymbol{j}\left(\boldsymbol{N}_{\boldsymbol{u}} \mathbf{5}\right)$ & \# Samples & $\boldsymbol{\pi}_{\boldsymbol{j}}$ & Current [A] \\
\hline 1 & 184 & 0.3673 & 3.0923 \\
\hline 2 & 82 & 0.1637 & 3.7050 \\
\hline 3 & 58 & 0.1158 & 4.3177 \\
\hline 4 & 115 & 0.2295 & 4.9303 \\
\hline 5 & 62 & 0.1238 & 5.5430 \\
\hline $\begin{array}{c}\text { Weighted } \\
\text { Average }\end{array}$ & 501 & 1 & 4.0596 \\
\hline
\end{tabular}

Table 2: Empirical distributions that define the weights $\pi_{j}$ for five future battery discharge profiles in a particlefiltering-based prognosis approach.

\begin{tabular}{|c|c|c|c|}
\hline $\boldsymbol{j}\left(\boldsymbol{N}_{\boldsymbol{u}}=\mathbf{7}\right)$ & \# Samples & $\boldsymbol{\pi}_{\boldsymbol{j}}$ & Current [A] \\
\hline 1 & 171 & 0.3413 & 3.0048 \\
\hline 2 & 42 & 0.0838 & 3.4424 \\
\hline 3 & 71 & 0.1417 & 3.8800 \\
\hline 4 & 31 & 0.0619 & 4.3177 \\
\hline 5 & 78 & 0.1557 & 4.7553 \\
\hline 6 & 57 & 0.1138 & 5.1929 \\
\hline 7 & 51 & 0.1018 & 5.6305 \\
\hline $\begin{array}{c}\text { Weighted } \\
\text { Average }\end{array}$ & 501 & 1 & 4.0355 \\
\hline
\end{tabular}

Table 3: Empirical distributions that define the weights $\pi_{j}$ for seven future battery discharge profiles in a particlefiltering-based prognosis approach.

Considering the aforementioned information, it was determined that the proposed approach for uncertainty representation would only include three possible paths within the implementation of the particle-filtering-based EOD prognosis framework. Lithium-Ion battery data shown in Section III was used to validate this method, comparing its performance with respect to a classic PF-based prognostic implementation (Saha and Goebel, 2009; Orchard and Vachtsevanos, 2009) that assumed only constant discharge current (computed as the average of past battery current measurements) for future operation. 
Figure 4 and Figure 5 show the results obtained when using the classic PF-based prognostic approach at 920[sec] of operation (40 particles and using 25 realizations of the longterm prediction before computing the EOD PDF). On the one hand, Figure 4 illustrates the results of the filtering and prognostic stages, showing that indeed an implementation based on model (5)-(7) can be used to quantify the effect that random changes in the battery discharge current have on the voltage of the device. Moreover, the predicted battery voltage includes information about the effect of the future evolution of the battery SOC in time, exhibiting an exponential drop as the SOC decreases.

Similar conclusions can be obtained from Figure 5, where the prediction stage is emphasized. The resulting EOD PDF allows building a 95\% confidence interval that provides information about when the battery would discharge if the future operating condition of the device is kept invariant.

Although this interval has a relative high precision (which is expected since there is no uncertainty associated to the future operating profile), it is important to mention that the bias associated to the assumed operation profile translated into overestimating the EOD time. Indeed, the computed expectation of the EOD time is 2224.91 [sec], while the ground truth discharge time occurred at 2123.7 [sec] of operation (101.21 [sec] before the expected value). A quick analysis of this information indicates that the proposed model for SOC estimation allows to efficiently incorporate measurement data and to generate reliable predictions paths; however, it must be said the high precision of the resulting PDF is only due to the fact that the uncertainty associated to the state estimates is bounded (the filter "learns" from data, thus the more extended the filtering stage is, the better the estimates) and because the uncertainty associated to the future battery use is neglected.

That is not necessarily true if the actual usage pattern of the battery is studied in detail. Figure 6 shows the obtained results when incorporating information from a statistical characterization, using the method proposed in Section 3 and Table 1. The fact of incorporating more uncertainty in the long-term predictions clearly has an impact on the precision of the resulting 95\% confidence interval; however, as a trade-off, the accuracy of the EOD expectation (2137.5 [sec]) is highly increased (see Figure 7) to a point where the algorithm has an error of only 13.8 [sec] in a 1203.7 [sec] prediction window. Although at a first glance many researchers could feel tempted to indicate that the precision of the proposed approach is disappointing, one must remember that a good prognostic algorithm should correctly characterize all uncertainty sources. As it has been already mentioned, by neglecting the uncertainty associated to the usage profile it is obvious that the resulting EOD PDF will be more "precise". The real question is if the computed precision represents the manner in which the user is operating the actual system.

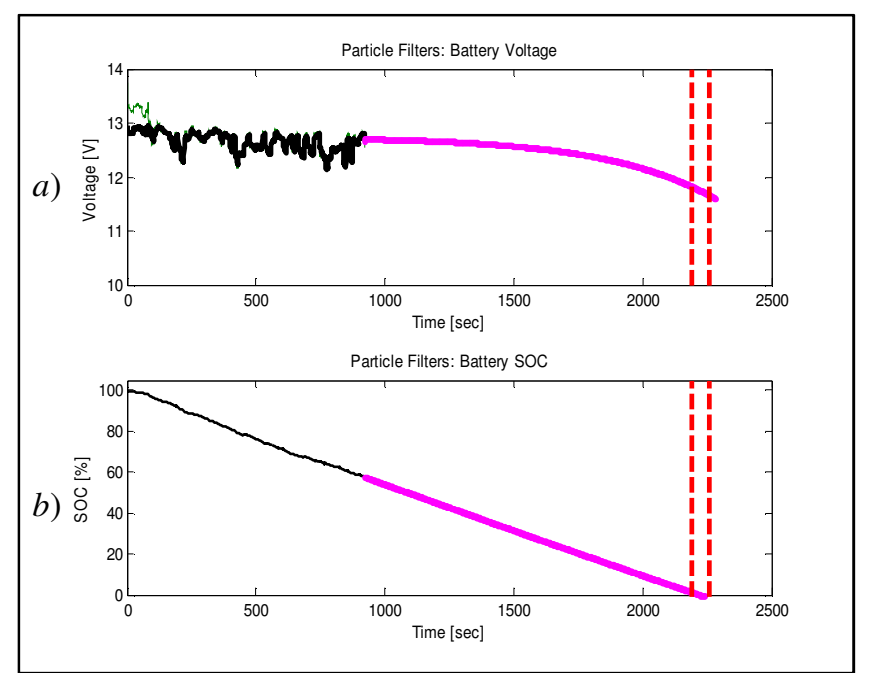

Figure 4: SOC prognosis assuming that the future discharge profile is a constant current equal to the average value measured in past data. (a) Measured voltage (thin green line), estimated voltage (dark black line), predicted voltage drop (magenta dashed line) and 95\% confidence interval for EOD (dashed vertical lines). (b) Estimated SOC [\%] (thin black line), predicted SOC [\%] (magenta thick line), and 95\% confidence interval for EOD (dashed vertical lines).

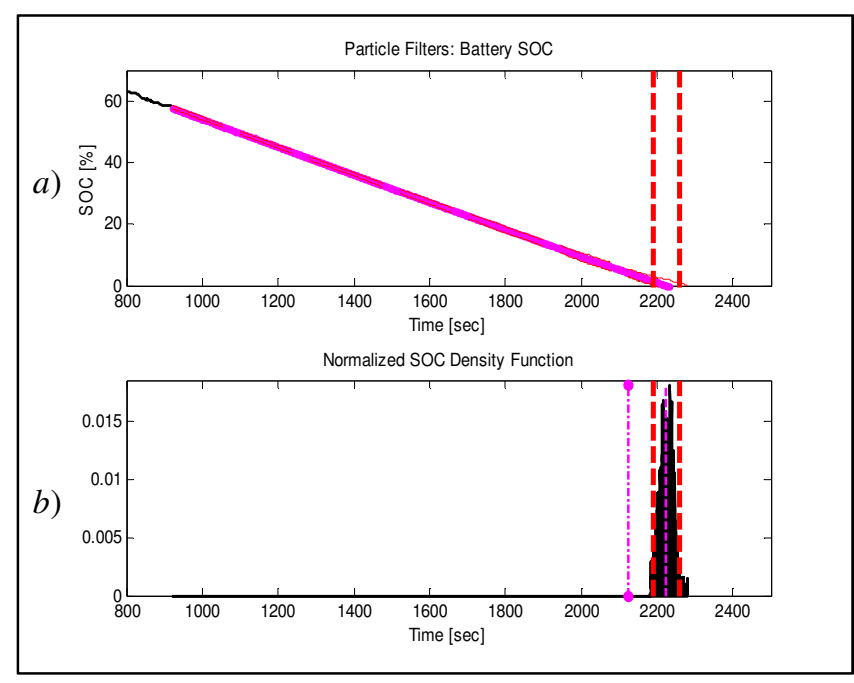

Figure 5: SOC prognosis assuming that the future discharge profile is a constant current equal to the average value measured in past data (zoom-in). (a) Estimated SOC [\%] (thin black line), predicted SOC [\%] (magenta thick line), 95\% confidence interval for predicted SOC [\%] (magenta thin lines)), and 95\% confidence interval for EOD (dashed vertical lines). (b) Ground truth EOD (vertical magenta segment with markers), predicted EOD PDF, and 95\% confidence interval for EOD.

A correct characterization of the tails of the PDF enable the implementation of much more sophisticated decisionmaking strategies, based on concepts such as the Just-in- 
Time point (JITP) instead of the expectation of the distribution, with the purpose of avoiding failures in the system that could occur before the moment that is being predicted by the prognosis module.

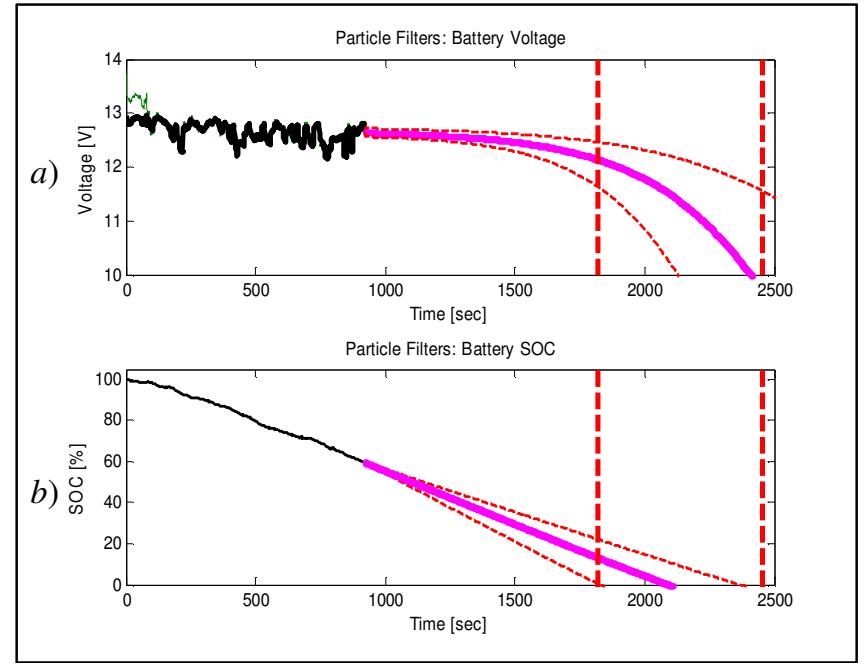

Figure 6: SOC prognosis assuming statistical

characterization of future discharge profile. (a) Measured voltage (thin green line), estimated voltage (dark black line), predicted voltage drop (magenta dashed line) and 95\% confidence interval for EOD (dashed vertical lines). (b) Estimated SOC [\%] (thin black line), predicted SOC [\%] (magenta thick line), and 95\% confidence interval for EOD (dashed vertical lines).

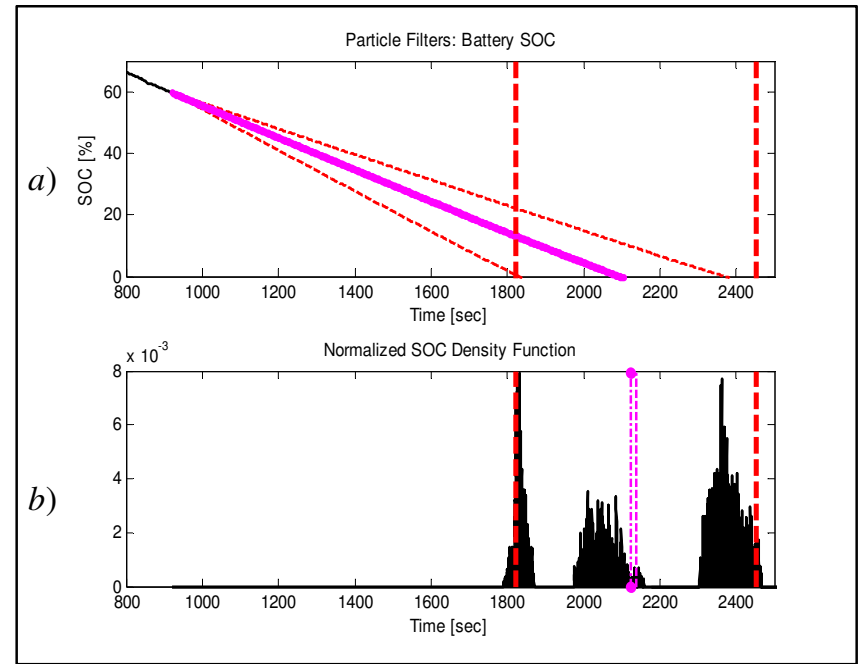

Figure 7: SOC prognosis assuming statistical characterization of future discharge profile. (zoom-in). (a) Estimated SOC [\%] (thin black line), predicted SOC [\%] (magenta thick line), 95\% confidence interval for predicted SOC [\%] (magenta thin lines)), and 95\% confidence interval for EOD (dashed vertical lines). (b) Ground truth EOD (vertical magenta segment with markers), predicted EOD $\mathrm{PDF}$, and $95 \%$ confidence interval for EOD.
One final remark can be made in term of the accuracy of the proposed algorithm if ad-hoc performance measures are to be used. In particular, Figure 8 shows the results obtained when using the " $\alpha-\lambda$ accuracy index" (Saxena et al., 2010) $(\alpha=15 \% ; \lambda=0.5)$. This measure determines if the predicted EOD is within a range defined by $\pm \alpha \%$ with respect to the true remaining time of operation, considering that the prediction window represents a fraction $\lambda$ of the total time of operation. If the system were to be timeinvariant, the remaining time should decrease linearly with slope equal to -1 . Although this assumption does not necessarily characterize the true evolution of the autonomy of the system in this case, it still represents a good indicator on the consistency of the prognostic result. Figure 8 shows that the conditional expectation of the EOD consistently decreases as more data is acquired, a fact that is important to validate the proposed algorithm since Figure 6 and Figure 7 illustrate only the response for a unique prediction time (920 [sec]).

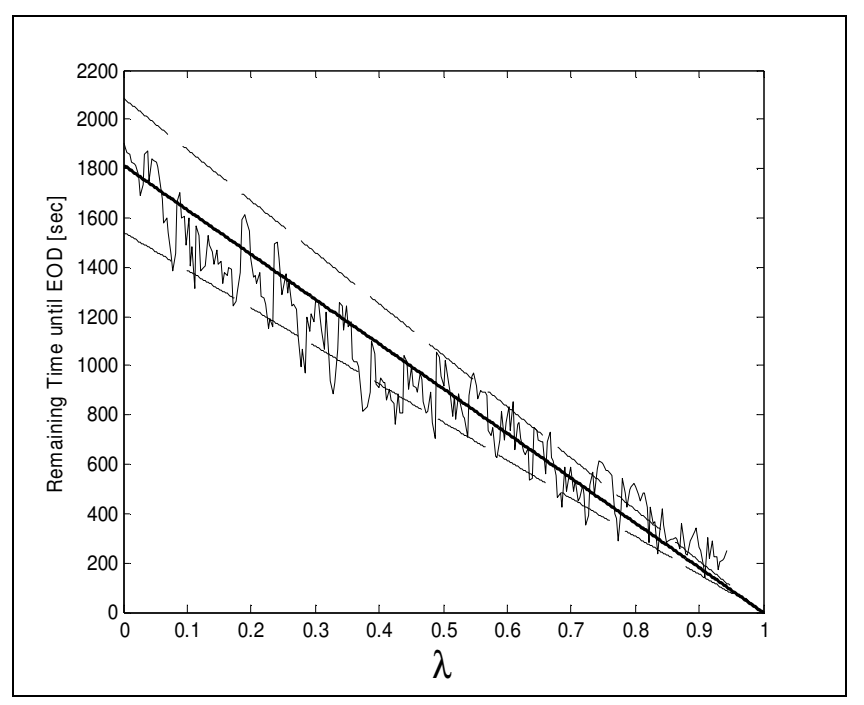

Figure 8: Prognosis performance evaluation based on the $\alpha$ $\lambda$ accuracy measure; $\alpha=15 \%$ and $\lambda=0.5$.

\section{CONClusion}

This paper presents the implementation of a particlefiltering-based prognostic framework that allows estimating the state-of-charge (SOC) and predicting the discharge time of energy storage devices, and more specifically lithium-ion batteries. The proposed approach uses an empirical statespace model inspired in the battery phenomenology and particle-filtering to study the evolution of the SOC in time; adapting the value of unknown model parameters during the filtering stage and enabling fast convergence for the state estimates that define the initial condition for the prognosis stage. SOC prognosis is implemented using a particlefiltering-based framework that considers uncertainty in the future discharge profile. The fact of incorporating more uncertainty in the long-term predictions clearly has an 
impact on the precision of the resulting 95\% confidence interval; however, as a trade-off, the accuracy of the EOD expectation (2137.5 [sec]) is highly increased (see Figure 7) to a point where the algorithm has an error of only 13.8 [sec] in a 1203.7 [sec] prediction window. Although the precision of the proposed algorithm is much worse that in the case when constant discharge current is assumed, it characterize in a better manner all uncertainty sources and the tails of the PDF; enabling the implementation of much more sophisticated decision-making strategies, based on concepts such as the Just-in-Time point (JITP).

\section{ACKNOWLEDGEMENT}

The authors want to thank CONICYT for the financial support provided through project FONDECYT \#1110070.

\section{REFERENCES}

Saha, B. and Goebel, K., (2009). "Modeling Li-ion Battery Capacity Depletion in a Particle Filtering Framework," Annual Conference of the Prognostics and Health Management Society, San Diego, CA.

Ranjbar, A.H., Banaei, A., Khoobroo, A., Fahimi, B., (2012). "Online Estimation of State of Charge in Li-Ion Batteries Using Impulse Response Concept," Smart Grid, IEEE Transactions on , Vol. 3, No.1, pp.360-367.

Pattipati, B., Sankavaram, C., and Pattipati, K., (2011). "System Identification and Estimation Framework for Pivotal Automotive Battery Management System Characteristics," Systems, Man, and Cybernetics, Part C: Applications and Reviews, IEEE Transactions on, Vol.41, No.6, pp.869-884.

Orchard, M., and Vachtsevanos, G., (2009). "A Particle Filtering Approach for On-Line Fault Diagnosis and Failure Prognosis," Transactions of the Institute of Measurement and Control, vol. 31, no. 3-4, pp. 221246.

Salkind, A.J., Fennie, C., Singh, P., Atwater, T., Reisner, D.E., (1999). "Determination of state-of-charge and state-of-health of batteries by fuzzy logic methodology," Journal of Power Sources, Vol. 80, Issue 1-2, pp. 293-300.

Charkhgard, M., and Farrokhi, M., (2010). "State-of-Charge Estimation for Lithium-Ion Batteries Using Neural Networks and EKF," Industrial Electronics, IEEE Transactions on, Vol.57, No.12, pp.4178-4187.

Vinh Do, D., Forgez, C., El Kadri Benkara, K., Friedrich, G., (2009). "Impedance Observer for a Li-Ion Battery Using Kalman Filter," Vehicular Technology, IEEE Transactions on, Vol.58, No.8, pp. 3930-3937.

Ran, L., Junfeng, W., Haiying, W., Gechen, L., (2010). "Prediction of state of charge of Lithium-ion rechargeable battery with electrochemical impedance spectroscopy theory," Industrial Electronics and Applications (ICIEA), 2010 the 5th IEEE Conference on, Vol., No., pp.684-688.
Cadar, D.V., Petreus, D.M., Orian, C.A., (2009). “A method of determining a lithium-ion battery's state of charge," 15th International Symposium for Design and Technology of Electronics Packages (SIITME) 2009, pp.257-260.

Qingsheng, S., Chenghui, Z., Naxin, C., Xiaoping, Z., (2010). "Battery State-Of-Charge estimation in Electric Vehicle using Elman neural network method," 29th Chinese Control Conference (CCC) 2010, pp. 59996003.

Di Z., Yan, M., Qing-Wen, B., (2011). "Estimation of Lithium-ion battery state of charge," 30th Chinese Control Conference (CCC) 2011, pp. 6256-6260.

Tang, X., Mao, X., Lin, J., Koch, B., (2011). "Li-ion battery parameter estimation for state of charge," American Control Conference (ACC) 2011, Vol., No., pp. 941946.

Saha, B., Goebel, K., Poll, S., Christophersen, J., (2009). "Prognostics Methods for Battery Health Monitoring Using a Bayesian Framework," IEEE Transactions on Instrumentation and Measurement, Vol.58, No.2, pp. 291-296.

Dalal, M., Ma, J., and He, D., (2011). "Lithium-ion battery life prognostic health management system using particle filtering framework," Proceedings of the Institution of Mechanical Engineers, Part O: Journal of Risk and Reliability, 225: 81-90.

Lee S., Kim J., Lee J., and Cho B.H., (2011). "Discrimination of Li-ion batteries based on Hamming network using discharging-charging voltage pattern recognition for improved state-of-charge estimation," Journal of Power Sources, v196, n4, pp. 2227-2240.

Santhanagopalan S., and White R.E., (2010). "State of charge estimation using an unscented filter for high power lithium ion cells," International Journal of Energy Research, v34, n2, pp. 152-163.

Hu C., Youn B.D., and Chung J., (2012). "A multiscale framework with extended Kalman filter for lithium-ion battery SOC and capacity estimation," Applied Energy, v92, pp. 694-704.

He Y., Liu X.T., Zhang C.B., Chen Z.H., (2013). “A new model for State-of-Charge (SOC) estimation for highpower Li-ion batteries", Applied Energy, v101, pp. 808814.

Orchard, M., Tang, L., Saha, B., Goebel, K., and Vachtsevanos, G., (2010) "Risk-Sensitive ParticleFiltering-based Prognosis Framework for Estimation of Remaining Useful Life in Energy Storage Devices," Studies in Informatics and Control, Vol. 19, Issue 3, pp. 209-218.

Arulampalam, M., Maskell, S., Gordon, N., and Clapp, T., (2002). "A Tutorial on Particle Filters for Online Nonlinear/Non-Gaussian Bayesian Tracking,", IEEE Transactions on Signal Processing, Vol. 50. 
Andrieu C., Doucet A., Punskaya E., (2001). "Sequential Monte Carlo Methods for Optimal Filtering," in Sequential Monte Carlo Methods in Practice, A. Doucet, N. de Freitas, and N. Gordon, Eds. NY: Springer-Verlag.

Doucet A., de Freitas N., Gordon N., (2001). "An introduction to Sequential Monte Carlo methods," in Sequential Monte Carlo Methods in Practice, A. Doucet, N. de Freitas, and N. Gordon, Eds. NY: Springer-Verlag.

Engel, S.J., Gilmartin, B.J., Bongort, K., Hess, A., (2000). "Prognostics, the real issues involved with predicting life remaining," Aerospace Conference Proceedings, 2000 IEEE, Vol.6, pp.457-469.

Orchard, M., Tobar, F., Vachtsevanos, G., (2009) “Outer Feedback Correction Loops in Particle Filtering-based Prognostic Algorithms: Statistical Performance Comparison," Studies in Informatics and Control, vol. 18, Issue 4, pp. 295-304.

Edwards, D., Orchard, M., Tang, L., Goebel, K., Vachtsevanos, G., (2010) "Impact of Input Uncertainty on Failure Prognostic Algorithms: Extending the Remaining Useful Life of Nonlinear Systems," Annual Conference of the Prognostics and Health Management Society 2010, Portland, OR, USA.

Chen, C. Vachtsevanos, G., Orchard, M., (2011) "Machine Condition Prediction Based on Adaptive Neuro-Fuzzy and High-Order Particle Filtering," IEEE Transactions on Industrial Electronics, vol. 58, no. 9, pp. 4353-4364.

Zhang, B., Sconyers, C., Byington, C., Patrick, R., Orchard, M., and Vachtsevanos, G., (2011). "A Probabilistic Fault Detection Approach: Application to Bearing Fault Detection," IEEE Transactions on Industrial Electronics, Vol. 58, No. 5, pp. 2011-2018.

Saxena, A., Celaya, J., Saha, B., Saha, S., Goebel, K., (2010). "Evaluating prognostics performance for algorithms incorporating uncertainty estimates," Aerospace Conference, 2010 IEEE, pp.1-11.

Tang, L., Orchard, M.E., Goebel, K., Vachtsevanos, G., (2011). "Novel metrics and methodologies for the verification and validation of prognostic algorithms," Aerospace Conference, 2011 IEEE, pp.1-8.

Gonzalez G.D., Orchard, M., Cerda J.L., Casali A. and Vallebuona, G., (2003). "Local models for soft-sensors in a rougher flotation bank," Minerals Engineering, vol. 16, no.5, pp. 441-453.

Hunter, J. S., (1986). "The exponentially weighted moving average," J. Qual. Technol., Vol. 18, pp. 203-209.

\section{BIOGRAPHIES}

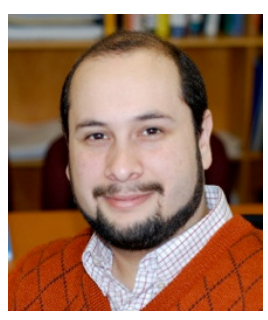

Dr. Marcos E. Orchard is Assistant Professor with the Department of Electrical Engineering at Universidad de Chile and was part of the Intelligent Control Systems Laboratory at The Georgia Institute of Technology. His current research interest is the design, implementation and testing of real-time frameworks for fault diagnosis and failure prognosis, with applications to battery management systems, mining industry, and finance. His fields of expertise include statistical process monitoring, parametric/non-parametric modeling, and system identification. His research work at the Georgia Institute of Technology was the foundation of novel real-time fault diagnosis and failure prognosis approaches based on particle filtering algorithms. He received his $\mathrm{Ph} . \mathrm{D}$. and M.S. degrees from The Georgia Institute of Technology, Atlanta, GA, in 2005 and 2007, respectively. He received his B.S. degree (1999) and a Civil Industrial Engineering degree with Electrical Major (2001) from Catholic University of Chile. Dr. Orchard has published more than 50 papers in his areas of expertise.

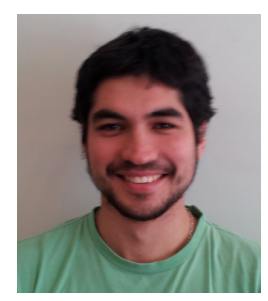

M.S. Matías A. Cerda was born in Santiago, Chile in 1987. He received both his B.Sc. and M.S. degrees in Electrical Engineering (2011) from Universidad de Chile. He currently is a Research Engineer at the "Lithium Innovation Center" (Santiago, Chile). His research interests include prognostics and health management for energy storage devices based on optimal and suboptimal Bayesian algorithms.

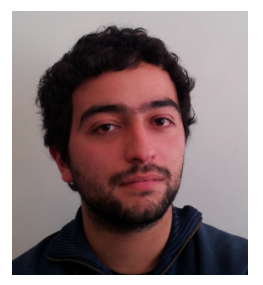

M.S. Benjamín E. Olivares was born in Santiago, Chile in 1987. He received both his B.Sc. and M.S. degrees in Electrical Engineering (2011) from Universidad de Chile. He currently is a Research Engineer at the "Lithium Innovation Center" (Santiago, Chile). His research interests include prognostics and health management for energy storage devices based on optimal and suboptimal Bayesian algorithms.

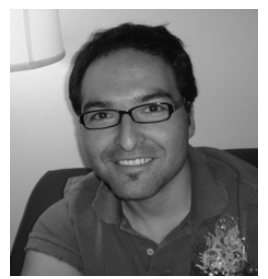

Dr. Jorge F. Silva is Assistant Professor at the Department of Electrical Engineering, University of Chile, Santiago, Chile. He received the Master of Science (2005) and Ph.D. (2008) in Electrical Engineering from the University of Southern California (USC). $\mathrm{He}$ is IEEE member of the Signal Processing and Information Theory Societies and he has participated as a 
reviewer in various IEEE journals on Signal Processing. Jorge F. Silva is recipient of the Outstanding Thesis Award 2009 for Theoretical Research of the Viterbi School of Engineering, the Viterbi Doctoral Fellowship 2007-2008 and Simon Ramo Scholarship 2007-2008 at USC. His research interests include: non-parametric learning; sparse signal representations, statistical learning; universal source coding; sequential decision and estimation; distributive learning and sensor networks. 